\title{
Subcycle laser control and quantum interferences in attosecond photoabsorption of neon
}

\author{
Xiaowei Wang, ${ }^{1,2}$ Michael Chini, ${ }^{2}$ Yan Cheng, ${ }^{2}$ Yi Wu, ${ }^{2}$ Xiao-Min Tong, ${ }^{3,4}$ and Zenghu Chang ${ }^{2, *}$ \\ ${ }^{1}$ Department of Physics, National University of Defense Technology, Changsha, Hunan 410073, China \\ ${ }^{2}$ CREOL and Department of Physics, University of Central Florida, Orlando, Florida 32816, USA \\ ${ }^{3}$ Division of Materials Science, Faculty of Pure and Applied Sciences, University of Tsukuba, Tsukuba, Ibaraki 305-8573, Japan \\ ${ }^{4}$ Center for Computational Sciences, University of Tsukuba, Tsukuba, Ibaraki 305-8577, Japan
}

(Received 9 April 2013; published 17 June 2013)

\begin{abstract}
The dynamics of an atom in a strong infrared laser field $\left(10^{13} \mathrm{~W} / \mathrm{cm}^{2}\right)$ result in substantial changes to the field-free electronic energy levels, which can be probed on time scales shorter than the laser cycle using isolated attosecond pulses. Here, we measure the transient absorption of an isolated attosecond pulse by laser-dressed bound states of neon near the first ionization threshold. The observed subcycle changes in the absorption spectrum result from both laser-induced ac Stark shifts and from quantum interferences between different multiphoton excitation pathways. We further demonstrate the ability to experimentally turn off the quantum interference mechanism by eliminating one of the interfering pathways.
\end{abstract}

DOI: 10.1103/PhysRevA.87.063413

PACS number(s): 32.80.Qk, 78.47.jb, 42.50.Hz

\section{INTRODUCTION}

Photoabsorption has long been used for spectroscopic study of atomic and molecular structure and for remote identification of chemical species. With the advent of ultrafast laser pulses, time-resolved transient absorption spectroscopy made it possible to study molecular processes on the $\sim 10$-fs time scale [1]. In transient absorption spectroscopy, a relatively weak probe laser pulse is used to monitor the changes in the absorption spectrum induced by a strong pump pulse with a variable time delay. Recently, the development and application of isolated attosecond pulses [2] has allowed probing of the atom with unprecedented time resolution, and attosecond transient absorption spectroscopy (ATAS) has been demonstrated to time-resolve electron dynamics on the few-femtosecond [3-6] and even suboptical-cycle [7-9] time scales.

Full numerical simulation and intuitive physical understanding of ATAS experiments has so far proved difficult. For this reason, most experiments were interpreted more generally, using a three-level atom [4,10-13] or other model systems [7] to explain the dominant features of the experiments. For recent ATAS experiments in bound states of helium [14-16], however, ab initio solutions of the time-dependent Schrödinger equation (TDSE) have also been obtained [17], which have helped to unify the existing theory and to demystify several previously unexplained features. However, helium is still a relatively simple (three-body) system, and it is unclear whether the theoretical models can be applied to more complex targets which are not exactly solvable. Therefore, before ATAS can be applied to molecular systems or condensed matter, further experiments on many-electron atomic systems are needed in order to test the current theoretical models. In this work, we extended the attosecond transient absorption technique to the neon atom, which contains ten electrons, and which can be solved numerically only by using a model potential in the single-active-electron (SAE) approximation. With neon as the absorption gas, we observed large laser-induced ac Stark shifts, laser-induced absorption structures corresponding to

\footnotetext{
*Zenghu.Chang@ucf.edu
}

multiphoton excitation of dipole-forbidden transitions, and subcycle oscillations of the absorption strength, which in most cases can be explained using the same theoretical description as in helium ATAS experiments. These experimental features can also be reproduced quite well by numerical calculation of the TDSE under the SAE approximation. Additionally, we confirmed by both experiment and calculation that the subcycle oscillations of the absorption strength result from quantum interference between two distinct pathways of excitation to a final state.

\section{EXPERIMENT}

The experiments were carried out on the same ATAS setup base that was used previously by our group [15]. The single isolated attosecond pulses (SIAPs) with pulse duration of $\sim 200$ as were generated by the interaction of 4-6 fs near-infrared (NIR) laser pulses centered at $730 \mathrm{~nm}$ (photon energy $\omega_{L} \approx 1.7 \mathrm{eV}$ ) with a xenon-filled gas cell by using the generalized double-optical-gating (GDOG) technique [18]. After being separated from the residual NIR laser by a $200-\mathrm{nm}$ aluminum filter, the SIAPs were focused by a toroidal mirror into a second neon-filled gas cell with inner diameter of $1.5 \mathrm{~mm}$. A hole-drilled mirror was placed between the toroidal mirror and the second gas cell to combine a time-delayed replica of the driving NIR pulse with the SIAP. After the absorption cell, the transmitted portion of the SIAP was sent to a home-built XUV spectrometer [19], in which the SIAPs were dispersed by a flat-field grating and detected by a microchannel plate (MCP)-phosphor detector which was imaged onto a thermoelectrically cooled CCD. The XUV spectrometer resolution was better than $50 \mathrm{meV}$ in the energy range of interest, as determined by measuring the bound-state absorption lines near the ionization thresholds of neon, argon, and helium.

The absorption cell was backed with 40 Torr of neon gas to absorb $\sim 75 \%$ of the spectrum above the neon ionization threshold in the absence of the NIR laser. The intensity of the NIR laser was controlled by an iris and determined from the measured laser pulse energy, duration, and focal spot size. The delay between the NIR and SIAP was introduced by 


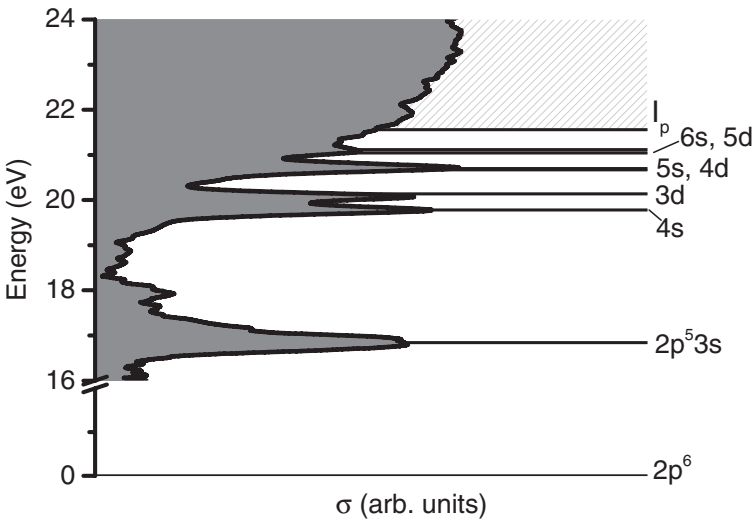

FIG. 1. Measured absorption cross section of neon atoms (black curve with gray filled area) and atomic energy levels of interest. The electron configurations are marked for each identified energy level.

a piezoelectric transducer (PZT), and a cw green laser was copropagated with both the SIAP and the delayed NIR laser to stabilize and control the delay [20] with a delay step size of 140 as and a stability of 50 as rms. At each delay step 10000 shots were accumulated to increase the signal-to-noise level. The carrier-envelope phase of the driving laser was of no importance in our measurement, since the SIAP generated using the GDOG technique is naturally synchronized to the field oscillation of the driving NIR pulse [21].

The ground state of neon can be described by the electron configuration $1 s^{2} 2 s^{2} 2 p^{6}$, and higher bound energy levels are formed by excitation of one valence electron resulting in a configuration of $1 s^{2} 2 s^{2} 2 p^{5} n l$. The energy levels of interest along with the measured absorption cross section are shown in Fig. 1. Due to dipole selection rules, absorption of an XUV photon can lead to excitation of a $2 p$ electron to the discrete $n s$ or $n d$ energy levels or to the $s$ or $d$ continua above the ionization threshold $I_{p}$, as indicated in Fig. 1. The introduction of a moderately intense NIR laser field, as in ATAS experiments, results in energy-level splitting and shifting, which can be observed through changes in the positions and shapes of the absorption features [17]. Therefore ATAS measurements enable us to resolve the evolution of laser-perturbed atomic energy levels on time scales comparable to or even shorter than the laser optical cycle.

Figure 2(a) shows the measured absorbance (proportional to the absorption cross section) of neon atoms perturbed by a $\sim 10 \mathrm{TW} / \mathrm{cm}^{2}$ laser field as a function of the time delay between the SIAP and the NIR field. For negative delays, where the SIAP arrives on the target before the NIR pulse, we can observe absorption lines corresponding to electron transitions from the ground state to excited states, as indicated in the figure. The broad continuous spectrum of the SIAP allows us to observe the absorption features corresponding to each bound state, as well as the above-threshold absorption, simultaneously, while the high resolution of the XUV spectrometer allows us to resolve the contribution of each state individually. As the delay between the two pulses approaches zero, the energy levels exhibit several interesting features. Firstly, the absorption cross section in the vicinity of the $1 s^{2} 2 s^{2} 2 p^{5} 3 s$ state oscillates with a period of half of the NIR cycle $\sim 1.3$ fs. Additionally, new laser-induced absorption
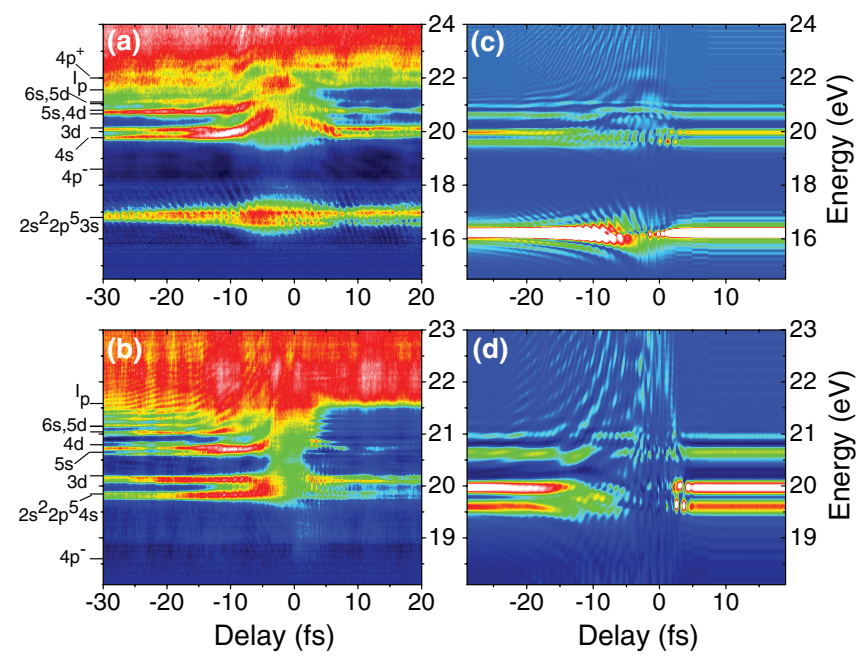

FIG. 2. (Color online) Measured delay-dependent absorbance spectra with a dressing laser intensity of (a) $\sim 10 \mathrm{TW} / \mathrm{cm}^{2}$ and (b) $\sim 75 \mathrm{TW} / \mathrm{cm}^{2}$. Panels (c) and (d) show the calculated absorption cross sections for dressing laser pulses $4 \mathrm{fs}$ in duration and with the same intensities as in panels (a) and (b).

structures are observed above the ionization threshold (near $22 \mathrm{eV}$ ) near zero delay. Finally, strong subcycle ac stark shifts are observed near zero delay for higher excited states, similar to those previously observed in helium atoms [7].

For negative delays, there is no clear observation of the ionization threshold, with a steplike increase in the absorption. Instead, we observe that the near-threshold absorption lines exhibit energy- and delay-dependent oscillations, appearing to shift above the ionization threshold as the delay approaches zero. The absorption edge associated with the ionization threshold appears only at positive delays. This feature was also observed in previous experiments in helium [15], and is likely because the near-threshold excited states of neon can be easily ionized by the delayed NIR field by singlephoton absorption. This process is remarkably similar to the perturbed free polarization decay previously observed in femtosecond transient absorption experiments [22] and in the vicinity of the $1 s 2 p$ state of helium [14], which results in the formation of absorption sidebands with energy spacing inversely proportional to the time delay. In order to investigate this process in more detail, we increased the NIR laser intensity to $\sim 75 \mathrm{TW} / \mathrm{cm}^{2}$, so that the perturbation to the highly excited states is much stronger. The delay-dependent absorption spectrogram is shown in Fig. 2(b), wherein multiple absorption sidebands are observed between $\sim 21$ and $\sim 22.5 \mathrm{eV}$ in negative delay.

\section{THEORETICAL CALCULATIONS AND DISCUSSIONS}

The theoretical calculations were performed by solving the TDSE in the integral form [23] under the SAE approximation, with the model potential obtained from the density functional theory with self-interaction correction [24]. The Fourier transform of the autocorrelation function (overlap of the time-dependent wave function with the group state weighted by transition dipole) is proportional to the photoabsorption cross section as detailed in [25] for a given time delay and 
external field. With the obtained time-dependent wave function $\Psi(t)$, we calculated the photoabsorption cross section as

$$
\sigma(\omega)=\frac{4 \pi}{c S(\omega)}\left|\int_{-\infty}^{\infty}\langle\Psi(t=-\infty)|r| \Psi(t)\rangle e^{i\left(\omega+\epsilon_{g}\right) t} d t\right|,
$$

with $\omega$ the photon energy, $S(\omega)$ the Fourier component of SIAP, and $\epsilon_{g}$ the ground-state energy. Different from Ref. [25], here we do not need to average the autocorrelation function over one IR cycle, since short pulses were used in the experiment. In the simulation, 1000 radial grid points and 32 partial waves were used. The numerical convergence has been checked by varying simulation parameters. The calculated photoabsorption cross sections of neon as a function of the time delay are shown in Figs. 2(c) and 2(d) for NIR intensities of $10 \mathrm{TW} / \mathrm{cm}^{2}$ and $75 \mathrm{TW} / \mathrm{cm}^{2}$, respectively. All the prevalent features observed experimentally in Figs. 2(a) and 2(b) also appear in the calculated results. We note that the absorption lines corresponding to the excited states of neon are slightly shifted in energy from those in the experiment, which is due to the model potential used in the calculations. However, the behavior of each energy level is remarkably similar to that observed in the experiment.

Although many interacting states are present in both the experiment and calculation, the dominant features can be explained in terms of the interactions of relatively few states. For example, the origin of the half-cycle oscillation in the $1 s^{2} 2 s^{2} 2 p^{5} 3 s$ state $(16.8 \mathrm{eV})$, which extends to negative delays, is illustrated schematically in Fig. 3(a). Here, there are two distinct pathways for electronic transitions between the ground state and $3 s$ state. When the SIAP arrives on the target, the absorption of XUV photons within the spectrum of the SIAP can result in excitation of several $n s$ and $n d$ states, one of which is $3 s$. Additionally, the $3 d$ state $(20.1 \mathrm{eV})$, which is energetically separated from the $3 s$ state by $2 \omega_{L}$, can be populated. When the delayed NIR laser arrives, the $3 d$ state population can be transferred to the $3 s$ state by emission of two NIR photons, assisted by the intermediate $3 p$ state. These two pathways finally lead to interference in the delay-dependent absorption cross section. Because both of these pathways are allowed as long as the SIAP arrives on the target before the NIR laser, the interference oscillations in the measured absorption strength extend to large negative delays, as indicated by the black curve in Fig. 3(c). On the other hand, the laser-induced $4 p^{+}$absorption structure shown in Figs. 2(a) and 2(b) near $22 \mathrm{eV}$ is localized near zero delay. This is because this structure arises from a dipole-forbidden transition $(2 p \rightarrow 4 p)$, which cannot be populated by the attosecond pulse alone, as illustrated in Fig. 3(b). In the moderately intense NIR field, the $4 p$ state forms two Floquet-like sidebands separated from the $4 p$ by $\omega_{L}$, which are marked as $4 p^{+}$and $4 p^{-}$in Figs. 2 and 3. Therefore the $4 p$ state can be populated by simultaneous absorption of an XUV photon with energy $E_{4 p} \pm \omega_{L}$ and absorption or emission of an NIR photon, resulting in laser-induced absorption structures near zero delay. In both experimental and theoretical spectrograms, only the $4 p^{+}$structure could be observed, since $4 p^{-}(18.6 \mathrm{eV})$ is not close to any $n s$ or $n d$ bound states while $4 p^{+}$overlaps with the continuum states. The half-cycle oscillations in the absorption (a)

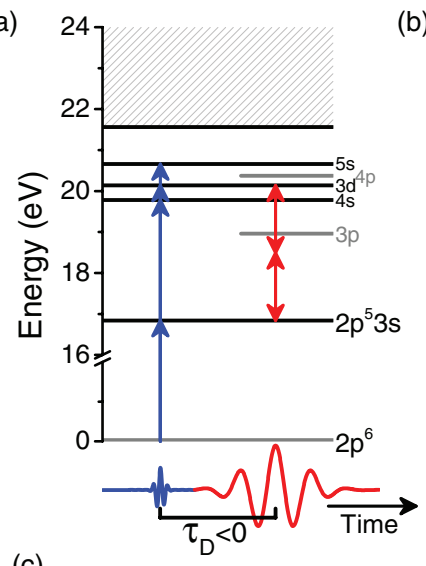

(b)

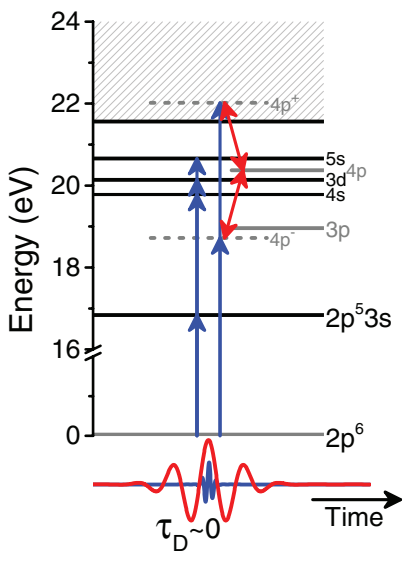

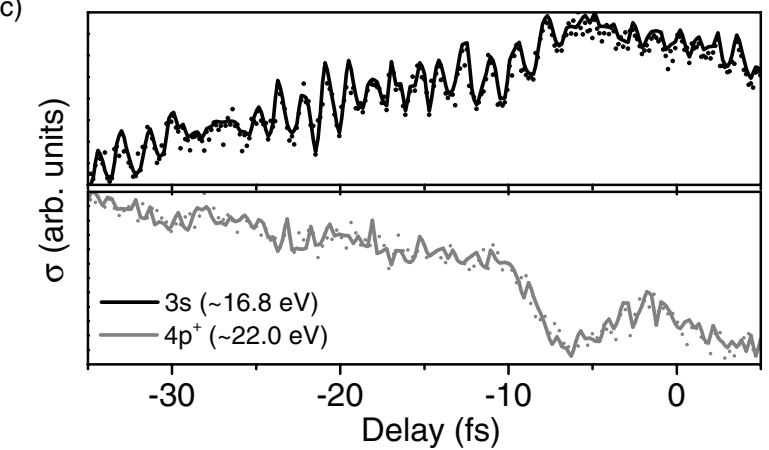

FIG. 3. (Color online) Diagram of direct and indirect pathways resulting in quantum interferences in the (a) $3 s$ and (b) $4 p^{+}$absorption lines. Energy levels indicated by black lines are accessible by one XUV photon absorption, while gray levels are forbidden by selection rules. Dashed gray energy levels in (b) indicate Floquet-like sidebands of the $4 p$ energy level. (c) Measured absorption cross sections in the vicinity of the $3 s$ (black) and $4 p^{+}$(gray) absorption lines. The dots indicate the experimentally obtained data points, whereas the solid lines are smoothed to show the major features. Whereas half-cycle ( $\sim 1.3 \mathrm{fs}$ ) oscillations can be observed in the $3 \mathrm{~s}$ absorption lines extending to large negative delays, oscillations in the $4 p^{+}$absorption can only be observed for delays between 0 and $-7 \mathrm{fs}$.

strength near the $4 p^{+}$structure, shown in the gray curve in Fig. 3(c), also result from interference between multiple quantum paths, since the $4 p^{+}$state can be reached either directly by absorption of one XUV photon or indirectly by initial excitation of the $4 p^{-}$state, followed by absorption of two NIR photons. Note that the Floquet-like sidebands exist only if the laser is present, so the light-induced structures as well as the corresponding interferences appear only near zero delay.

Previously, the role that a particular state plays in ATAS experiments was identified through the use of test calculations which dynamically eliminated the state during the time propagation of the TDSE $[14,15]$. The same method was adopted here to confirm the origin of a $4 p^{+}$structure by individually removing $3 p, 4 p$, and $4 f$ states in the test calculations. These calculations confirm that $4 p^{+}$absorption results from the laser-induced sideband of the $4 p$ state. We additionally adopt an experimental technique to confirm the origin of the interference in the vicinity of the $3 s$ state by suppressing the indirect pathway. In order to achieve this, 

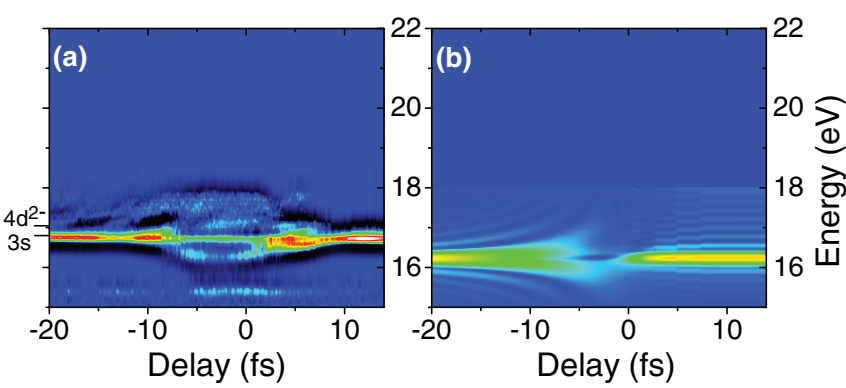

FIG. 4. (Color online) (a) Measured absorbance and (b) calculated absorption cross section as a function of the time delay, using isolated attosecond pulses filtered by an indium filter which allows transmission of XUV pulses within the spectrum range from $11 \mathrm{eV}$ to $17 \mathrm{eV}$. In this case, no delay-dependent quantum path interferences are observed.

the aluminum filter used to filter out the SIAPs was replaced with a 200-nm-thick indium filter, which allows transmission of XUV pulses only within the spectral range from 11 to $17 \mathrm{eV}$. With the indium filter, the indirect pathway involving the $3 d$ state is effectively turned of due to the lack of XUV spectral components above $17 \mathrm{eV}$. Instead, only the direct pathway for excitation of the $3 s(16.8-\mathrm{eV})$ state is possible. Figure 4(a) shows the measured ATAS spectrogram, in which no quantum interferences are observed, and the calculated cross section shown in Fig. 4(b) is in excellent agreement with the experiment result. This provides direct experimental evidence that the observed oscillations result from quantum interference between the $3 s$ and higher-lying excited states, such as the $3 d$, by the NIR laser. Instead, we observe only the energy-level splitting of the $3 s$ state near zero delay, similar to the Autler-Townes splitting observed in previous experiments in helium $[14,15]$.

\section{CONCLUSIONS}

In conclusion, we measured the transient absorption of an isolated attosecond pulse by laser-dressed bound states of neon atoms. The observed subcycle changes in the neon absorption spectrum result from both subcycle ac Stark shifts and from quantum interferences between different multiphoton excitation pathways. New laser-induced absorption structures corresponding to multiphoton excitation of dipole-forbidden transitions were also observed. Furthermore, we experimentally demonstrated the ability to turn off the quantum interference observed in the $3 s$ state by removing the indirect excitation pathway via the $3 d$ state. Although direct numerical simulation of the ten-electron neon atom is still impossible, we demonstrate that the dynamics can still be interpreted through TDSE simulations by relying on the SAE approximation and through relatively simple models involving few interacting states. The applicability of these existing theoretical models to the present neon ATAS experiments paves the way to extend the ATAS technique to more complex systems.

\section{ACKNOWLEDGMENTS}

This work is supported by the US Army Research Office and by the National Science Foundation under Grant No. 1068604. X.-M.T. was supported by a Grand-in-Aid for Scientific Research (C24540421) from the Japan Society for the Promotion of Science and the simulation was supported by the HA-PACS Project for advanced interdisciplinary computational sciences by exa-scale computing technology.
[1] R. W. Schoenlein, L. A. Peteanu, R. A. Mathies, and C. V. Shank, Science 18, 412 (1991).

[2] Z. Chang and P. Corkum, J. Opt. Soc. Am. B 27, B9 (2010).

[3] E. Goulielmakis, Z.-H. Loh, A. Wirth, R. Santra, N. Rohringer, V. S. Yakovlev, S. Zherebtsov, T. Pfeifer, A. M. Azzeer, M. F. Kling et al., Nature (London) 466, 739 (2010).

[4] H. Wang, M. Chini, S. Chen, C. H. Zhang, Y. Cheng, F. He, Y. Cheng, Y. Wu, U. Thumm, and Z. Chang, Phys. Rev. Lett. 105, 143002 (2010).

[5] Z. Loh, C. H. Greene, and S. R. Leone, Chem. Phys. 350, 7 (2008).

[6] M. Tarana and C. H. Greene, Phys. Rev. A 85, 013411 (2012).

[7] M. Chini, B. Zhao, H. Wang, Y. Cheng, S. X. Hu, and Z. Chang, Phys. Rev. Lett. 109, 073601 (2012).

[8] P. Ranitovic, X. M. Tong, C. W. Hogle, X. Zhou, Y. Liu, N. Toshima, M. M. Murnane, and H. C. Kapteyn, Phys. Rev. Lett. 106, 193008 (2011).

[9] M. Holler, F. Schapper, L. Gallmann, and U. Keller, Phys. Rev. Lett. 106, 123601 (2011).

[10] W. C. Chu and C. D. Lin, Phys. Rev. A 87, 013415 (2013).

[11] A. N. Pfeiffer and S. R. Leone, Phys. Rev. A 85, 053422 (2012).

[12] S. Pabst, A. Sytcheva, A. Moulet, A. Wirth, E. Goulielmakis, and R. Santra, Phys. Rev. A 86, 063411 (2012).

[13] J. C. Baggesen, E. Lindroth, and L. B. Madsen, Phys. Rev. A 85, 013415 (2012).
[14] S. Chen, M. J. Bell, A. R. Beck, H. Mashiko, M. Wu, A. N. Pfeiffer, M. B. Gaarde, D. M. Neumark, S. R. Leone, and K. J. Schafer, Phys. Rev. A 86, 063408 (2012).

[15] M. Chini, X. Wang, Y. Cheng, Y. Wu, D. Zhao, D. A. Telnov, S.-I. Chu, and Z. Chang, Sci. Rep. 3, 1105 (2013).

[16] L. Argenti, C. Ott, T. Pfeifer, and F. Martin, arXiv:1211.2566 (2012).

[17] S. Chen, M. Wu, M. B. Gaarde, and K. J. Schafer, Phys. Rev. A 87, 033408 (2013).

[18] X. Feng, S. Gilbertson, H. Mashiko, H. Wang, S. D. Khan, M. Chini, Y. Wu, K. Zhao, and Z. Chang, Phys. Rev. Lett. 103, 183901 (2009).

[19] X. Wang, M. Chini, Y. Cheng, Y. Wu, and Z. Chang, Appl. Opt. 52, 323 (2013).

[20] M. Chini et al., Opt. Express 17, 21459 (2009).

[21] S. Gilbertson, S. D. Khan, Y. Wu, M. Chini, and Z. Chang, Phys. Rev. Lett. 105, 093902 (2010).

[22] C. H. Brito-Cruz, J. P. Gordon, P. C. Becher, R. L. Fork, and C. V. Shank, IEEE J. Quantum Electron. 24, 261 (1988).

[23] X. M. Tong, K. Hino, and N. Toshima, Phys. Rev. A 74, 031405(R) (2006).

[24] X. M. Tong and Shih-I. Chu, Phys. Rev. A 55, 3406 (1997).

[25] X. M. Tong and N. Toshima, Phys. Rev. A 81, 063403 (2010). 